\title{
The s-process in stellar sites
}

\author{
Sergio Cristallo ${ }^{1,2, \star}$ \\ ${ }^{1}$ Osservatorio Astronomico d'Abruzzo, via Maggini, 64100 Teramo, Italy \\ ${ }^{2}$ Sezione INFN di Perugia, via Pascoli, 06123 Perugia, Italy
}

\begin{abstract}
Stars are marvellous caldrons where all the elements of the Universe (apart from hydrogen and helium) have been synthesized. The solar system chemical distribution is the result of many pollution episodes from already extinct stellar generations, occurred at different epochs before the Sun formation. Main nucleosynthesis channels responsible for the formation of heavy elements are the rapid neutron capture process (the r-process) and the slow neutron capture process (the s-process). Hereafter, I will describe the theory of the s-process and the stellar sites where it is active.
\end{abstract}

\section{Introduction}

The history of the chemical enrichment of the Universe follows a simple paradigm: helium and metals (i.e. elements with $A \geq 12$ ) increase at the expense of hydrogen. Starting with an hydrogen mass fraction of about 0.25 and no metals, the Universe evolved to the current $\sim 65 \%$ of $\mathrm{H}$ and $\sim 4 \%$ of metals characterizing star forming regions. The distribution of heavy elements $(A>56)$ in the solar system shows an exponential decline, together with clearly identified abundance peaks, related to the $\mathrm{s}$ (slow) and to the $\mathrm{r}$ (rapid) processes [1]. The abundances of heavy elements are far greater that can be reproduced in nuclear statistical equilibrium, so that they seem to require a non-equilibrium mechanism. Their synthesis cannot occur via charged particle reactions, due to the increasing Coulomb barrier with increasing atomic number. Moreover, ${ }^{56} \mathrm{Fe}$ is the most tightly bound nucleus and, therefore, neither fusion nor fission processes may release energy starting from it. Thus, the only way to efficiently produce isotopes heavier than iron is via neutron capture processes. In such a case, in fact, heavy elements can be easily created, even at extremely low energies (due to the fact that, in general, neutron capture cross sections increase with decreasing energy).

Multiple neutron captures on a nucleus (Z,A) occur if the new synthesized neutron-rich isotopes $(\mathrm{Z}, \mathrm{A}+\mathrm{i})$ are stable (with $\mathrm{i}$ ranging from 1 to 6$)$. On the contrary, if the nucleus $(\mathrm{Z}, \mathrm{A}+\mathrm{i})$ is radioactive, the question whether it will decay to its isobar $(Z+1, A+i)$ or will capture another neutron depends upon the relative lifetimes between the decay lifetime and the timescale of the neutron capture process. One might expect to find in stars a large spread of neutron fluxes, each one characterizing different evolutionary phases. On the contrary, the most common fluxes are either quite small (s-process: $\mathrm{n}_{n} \sim 10^{7} \mathrm{~cm}^{-3}$ ) or quite large (r-process: $\mathrm{n}_{n}>10^{20} \mathrm{~cm}^{-3}$ ). The $\mathrm{r}$ process is believed to occur during late phases of massive stars evolution, even if the exact site is still matter of debate (SuperNovae $[2,3]$ vs. Neutron stars mergers [4]). Due to the difficulties in modelling such a process, together

^e-mail: sergio.cristallo@inaf.it 


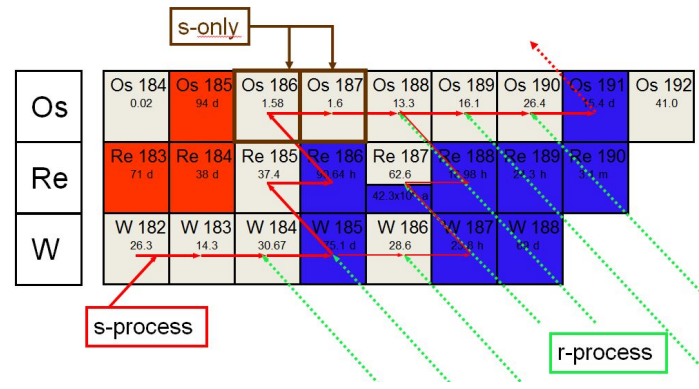

Figure 1. Scheme of the s-process pattern in the W-Re-Os region.

with the still large uncertainties affecting its nuclear inputs, the contribution to the Sun provided by the $r$-process is commonly estimated by subtracting that coming from the s-process $(r=1-s)$. A correct estimate of s-process yields is thus mandatory for a detailed knowledge of the chemical evolution of our Galaxy.

\section{The theory of the s-process}

A typical s-process pattern is reported in Figure 1. The Re-Os-W region is shown. Stable isotopes are colored in gray, while unstable isotopes are marked with different colors (red for those decaying $\beta^{+}$or capturing an electron; blue for those decaying $\beta^{-}$). Numbers in each box represent the solar isotopic fraction (for stable isotopes) and decay lifetimes (for the unstable ones). The main s-process flow is traced by red heavy arrows (horizontal lines are neutron captures, while slanting ones are $\beta^{-}$decays). In correspondence of unstable isotopes (e.g. ${ }^{185} \mathrm{~W}$ ), s-process branchings may appear and two different paths may develop. The predominance of the channel is determined by the ratio between the neutron capture cross section timescale and the decay lifetime. The contribution from the r-process is highlighted by dotted green arrows. Interestingly, there are isotope which are shielded by an r-process contribution by their stable isobars (see, for instance, the couples ${ }^{186} \mathrm{Os}_{-}{ }^{186} \mathrm{~W}$ and ${ }^{187} \mathrm{Os}-{ }^{187} \mathrm{Re}$ ). Those isotopes are named s-only. Their nucleosynthesis is of paramount importance to calibrate s-process calculations (see [17] and references therein). Main seeds for the s-process are ${ }^{56} \mathrm{Fe}$ nuclei. In Figure 2 the solar abundances for isotopes heavier than iron are shown (upper panel). Three peaks clearly emerge from the distribution, corresponding to nuclei with neutron magic nuclei $(\mathrm{N}=50, \mathrm{~N}=82$ and $\mathrm{N}=126$ ). Those isotopes are characterized by closed shells configurations and, thus, they are particularly stable with respect to their neighbors. This can be better understood looking at the lower panel of Figure 2, where neutron capture cross sections are reported (in mb). Among the isotopes with the slowest neutron capture cross sections there are ${ }^{88} \mathrm{Sr},{ }^{138} \mathrm{Ba}$ and ${ }^{208} \mathrm{~Pb}$. They characterize the three peaks of the s-process: $l s$ elements (Sr-Y-Zr), $h s$ elements (Ba-La-Ce-Pr-Nd) and lead $(\mathrm{Pb})$. The precise knowledge of their neutron capture cross sections is of paramount importance to determine the ratio between light and heavy s-process elements, the so called $[h s / l s]$ s-process index. For this reason, dedicated experimental campaigns are currently going on at the n_TOF facility at CERN [7]. At atomic weights far from the closed shells, the so-called local approximation holds, i.e. the product $\sigma \cdot \mathrm{n}$ is almost constant. In this case, the cross sections are so large that the differences between the products is much smaller than the magnitude of either one of them.

Another important quantity in s-process calculations is the neutron-to-seed ratio, i.e. the ratio between the available neutrons and the seeds from which the s-process start. Depending on this ratio, different s-process distributions can be obtained. In fact, the larger the number of available neutrons per iron seed, the larger the masses of the synthesized isotopes. For instance, at solar metallicity only $l s$ elements are efficiently produced, due to the very low neutron cross sections of ${ }^{88} \mathrm{Sr},{ }^{89} \mathrm{Y}$ and ${ }^{90} \mathrm{Zr}$, 


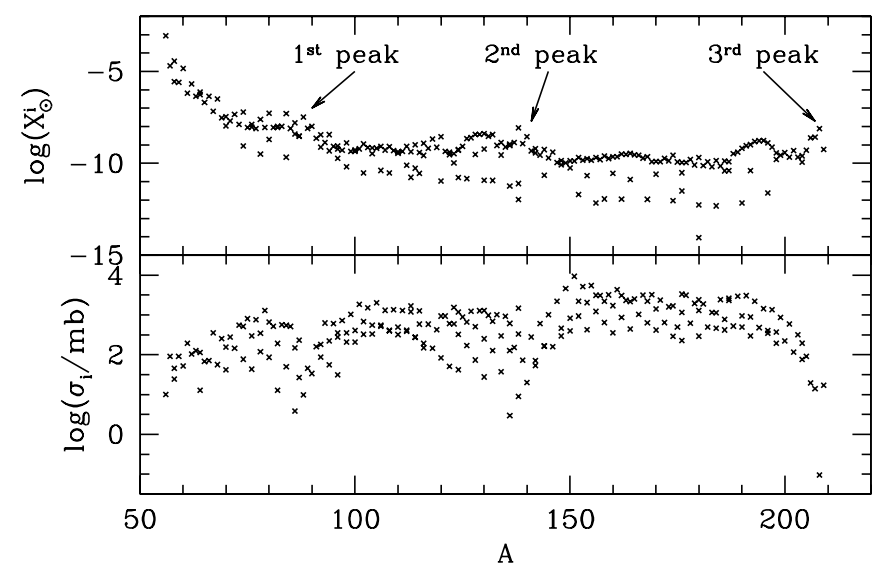

Figure 2. Heavy elements isotopic solar distribution (upper panel) and corresponding neutron capture cross sections (lower panel). Experimental data are mainly taken from [6].

which act as bottlenecks for the s-process path. If such a barrier is overtaken, the next one is encountered at larger atomic masses (i.e. for ${ }^{139} \mathrm{Ba},{ }^{139} \mathrm{La}$ and ${ }^{140} \mathrm{Ce}$ ). At low metallicities the s-process pattern goes beyond this second barrier and lead can be largely synthesized. The different s-process trends as a function of the initial metallicity are reported in the left panel of Figure 3.

Major neutron sources in stars are the ${ }^{13} \mathrm{C}(\alpha, \mathrm{n}){ }^{16} \mathrm{O}$ and the ${ }^{22} \mathrm{Ne}(\alpha, \mathrm{n}){ }^{25} \mathrm{Mg}$ reactions. While the number of iron seeds directly scales with metallicity, the number of neutrons may have a primary behavior, that is it may be a direct product of $\mathrm{H}$ - and He-burning. In such a way, different s-process distributions result for different initial metallicities. The first reaction dominates in low mass stars $\left(M<4 \mathrm{M}_{\odot}\right.$, see Section 3) and it is responsible for the main component of the s-process $(88 \leq \mathrm{A} \leq 208)$. The second, instead, is the main neutron producer in massive stars (see Section 4) and it accounts for the weak component of the s-process $(\mathrm{A}<88)$. Due to their importance in determining the efficiency of the sprocess nucleosynthesis, both reactions have been explored into detail in the past (see [10] and [11], respectively).

\section{The s-process in low and intermediate mass stars}

The s process is at work in Thermally Pulsing AGB (TP-AGB) phase of low mass stars $(1<\mathrm{M} /$ $\left.M_{\odot}<4 ;[12,13]\right)$. In those objects, freshly synthesized elements are carried out to the surface by means of recurrent mixing episodes, commonly known as Third Dredge Ups (TDUs) (see [9] and references therein; see also [14]). Interested readers may download TP-AGB surface abundances and yields from the FRUITY database ${ }^{1}[15,16]$. The structure of an AGB star is made of a partially degenerate carbon-oxygen core, an He shell separated from an $\mathrm{H}$ shell by a thin region, and by a convective envelope. The energy is provided for most of the time by the $\mathrm{H}$-shell, located below the H-rich envelope. Recurrently, the H-burning in the shell is interrupted by the growing up of thermonuclear runaways, triggered by violent He-burning ignitions (Thermal Pulses, TPs). During a TP, the region between the two shells becomes convective, while the layers above expand. As a consequence, the

\footnotetext{
${ }^{1}$ http://fruity.oa-teramo.inaf.it/
} 

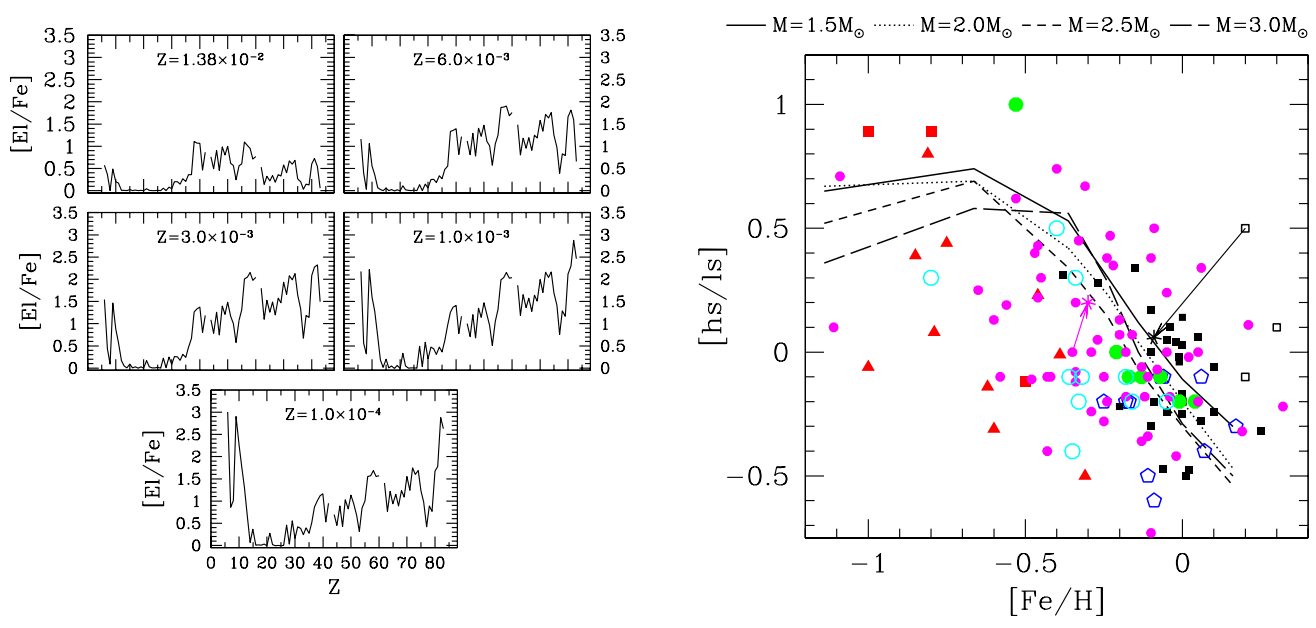

Figure 3. Left panel: s-process distributions at different metallicities for models with initial mass $M=2 \mathrm{M}_{\odot}$ (from [8]). Right panel: comparison between theory and observations at different metallicities (from [15]).

H-burning shell possibly switches off, allowing the convective envelope to penetrate in regions where heavy isotopes have been freshly synthesized. As already highlighted, the main neutron sources is the ${ }^{13} \mathrm{C}(\alpha, \mathrm{n}){ }^{16} \mathrm{O}$ reaction. In order to obtain a sufficient amount of ${ }^{13} \mathrm{C}$ for the activation of the s-process, protons from the envelope have to be mixed (with a low efficiency) with the ${ }^{12} \mathrm{C}$-rich material in the underlying radiative zone. Those protons are captured by ${ }^{12} \mathrm{C}$ and form ${ }^{13} \mathrm{C}$ via the nuclear chain ${ }^{12} \mathrm{C}(\mathrm{p}, \gamma){ }^{13} \mathrm{~N}\left(\beta^{-}\right){ }^{13} \mathrm{C}$. In such a way, a ${ }^{13} \mathrm{C}$-enriched layer, the so-called ${ }^{13} \mathrm{C}$-pocket, forms. The ${ }^{13} \mathrm{C}$ burns in radiative conditions between two TPs at temperatures around $100 \mathrm{MK}$. The attained neutron densities are $\sim 10^{7} \mathrm{~cm}^{-3}$. The physical process leading to the formation of the ${ }^{13} \mathrm{C}$ pocket is far from being understood. In the past, different mechanisms have been proposed, among which opacityinduced mixing [8], Kelvin-Helmholtz instabilities plus gravity waves [18] and magnetic buoyancy [20]. Other non-convective mixings, such as those induced by rotation (e.g. Eddington-Sweet circulations and Goldreich-Schubert-Fricke instabilities), have been considered by [19], who demonstrated their relevance in shaping the ${ }^{13} \mathrm{C}$ distribution in the pocket (with obvious consequences on the following s-process nucleosynthesis). In the left panel of Fig. 3 typical s-process distributions as a function of the initial metallicity are reported. The showed patterns, which have been obtained with the FUNS code $[8,9]$, highlight the strong dependence of s-process distributions from the initial iron content. In fact, as soon as the latter decreases, the abundances of $h s$ elements increase, followed by a rapid grow of the lead peak at very low metallicities. Note also the progressive growth with decreasing metallicities of the overabundances of carbon and fluorine. As a further demonstration of the key role of the neutron-to-seed ratio, we report in the right panel of Fig. 3 a comparison between theory and observations, where we plot the $[h s / l s]$ s-process index as a function of the initial iron content. 


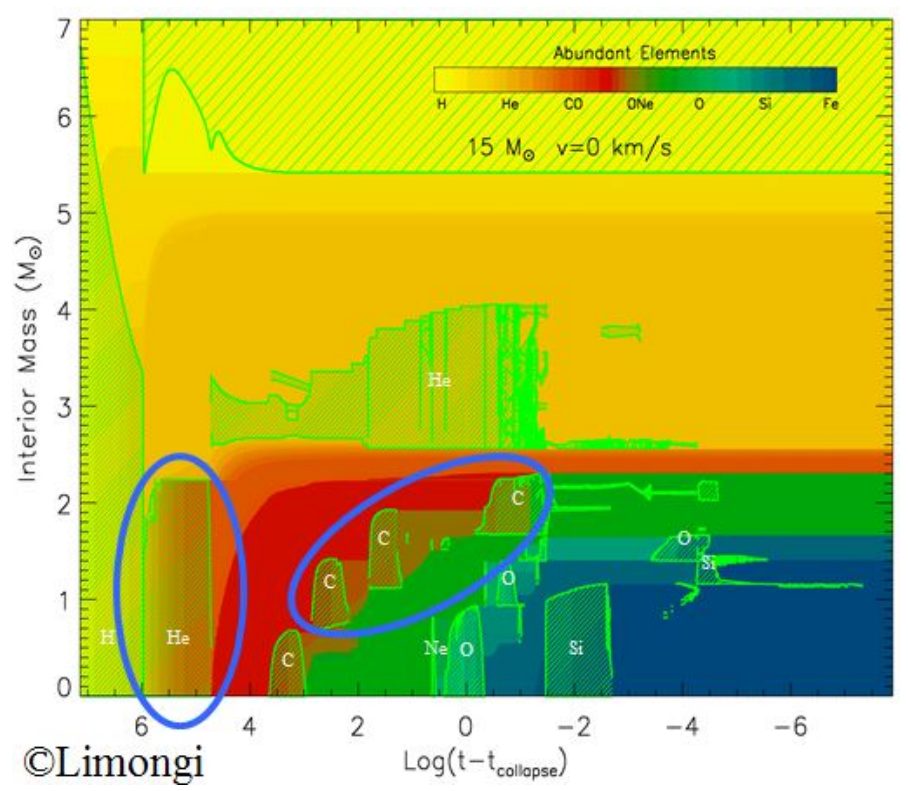

Figure 4. Kippenhahn diagram of a non rotating star with initial mass $M=15 M_{\odot}$ (copyright Marco Limongi).

Starting from the largest metallicities, it can be seen that the $[h s / l s]$ ratio increases with decreasing metallicities, proving that heavier elements (in this case the $h s$ ones) are produced. At $[\mathrm{Fe} / \mathrm{H}]=-0.7$ a plateau in the $[h s / l s]$ appears. This metallicity marks the region where lead starts being efficiently synthesized, due to the strong decrease of the neutron-to-seed ratio.

In low mass AGB stars, an additional (marginal) contribution comes from the ${ }^{22} \mathrm{Ne}(\alpha, \mathrm{n})^{25} \mathrm{Mg}$ reaction, which burns at higher temperatures (T 300MK) during TPs. During this episode, neutron densities are larger $\left(>10^{11} \mathrm{~cm}^{-3}\right)$, but the neutron exposure (that is the integrated exposure to neutron fluence) is definitely lower with respect to the radiative ${ }^{13} \mathrm{C}$ burning. This derives from the fact that the neutron irradiation lasts for a lower time interval (yrs compared to tens of thousands yrs). The ${ }^{22} \mathrm{Ne}$ forms during TPs via two $\alpha$ captures: ${ }^{14} \mathrm{~N}(\alpha, \gamma){ }^{18} \mathrm{~F}\left(\beta^{+}\right){ }^{18} \mathrm{O}(\alpha, \gamma){ }^{22} \mathrm{Ne}$. This source is dominant in more massive AGBs $\left(5<\mathrm{M} / \mathrm{M}_{\odot}<8\right)$, because temperatures largely exceed $300 \mathrm{MK}$ during TPs. Moreover, those objects have larger core masses and, therefore, the contribution from the ${ }^{13} \mathrm{C}$ is marginal [17]. Intermediate Mass AGB stars marginally contribute to the Galactic chemical evolution. Their role, instead, is of primary importance in the pollution history of s-rich galactic Globular Clusters (GCs), such as M22 [21] and M4 [22]. In fact, [23] (and independently [24]) demonstrated that AGB stars with masses between 3 and $6 \mathrm{M}_{\odot}$ have been the dominant polluters of those systems.

\section{The s-process in massive stars}

Elements between iron and the first s-process peak are efficiently synthesized by the weak s-process $[25,26]$. This nucleosynthesis process is at work during the core He-burning and the C-shell burning phases of massive $\left(M>9 M_{\odot}\right)$ stars evolution (see Figure 4). In both episodes, neutrons are provided by the abundant ${ }^{22} \mathrm{Ne}$, whose formation path is the same as in lower mass stars. During core Heburning, central temperatures exceeds $300 \mathrm{MK}$ and efficiently activate the ${ }^{22} \mathrm{Ne}(\alpha, \mathrm{n})^{25} \mathrm{Mg}$ reaction. This phase, lasting about $1 \mathrm{Myr}$ and characterized by rather low neutron densities $\left(\mathrm{n} \sim 10^{6} \mathrm{~cm}^{-3}\right)$, 
almost yields the final weak s-process abundances of the model. During core He-burning, ${ }^{22} \mathrm{Ne}$ is not completely burnt. Thus, additional neutrons are available during the following C-burning shell phase $^{2}$. In this phase, the $\alpha$ particles are provided by ${ }^{12} \mathrm{C}\left({ }^{12} \mathrm{C}, \alpha\right){ }^{20} \mathrm{Ne}$ reactions. The neutron exposure is roughly the same as during core He-burning. However, this evolutionary phase is definitely shorter $(\sim 1 \mathrm{kyr})$ and the temperature definitely higher $(\mathrm{T}>1 \mathrm{GK})$. This leads to very large neutron densities (exceeding $>10^{12} \mathrm{~cm}^{-3}$ ), but to a rather low neutron exposure. We refer to [27] for a detailed description of the nucleosynthesis and of the effects induced by variations of nuclear reaction rates.

Results are definitely different if the star is rotating. In that case, in fact, rotation induced mixing carries ${ }^{12} \mathrm{C}$ from the $\mathrm{He}$ convective core in the $\mathrm{H}$ burning shell, where it is transformed to ${ }^{14} \mathrm{~N}$ via $\mathrm{CNO}$ burning. This has deep consequences on ${ }^{22} \mathrm{Ne}$, whose abundance largely increases. The larger the initial rotation rate, the larger the ${ }^{22} \mathrm{Ne}$ production, with important consequences on the s-process production by massive stars at low metallicities [28].

\section{References}

[1] Burbidge, E. M. ,Burbidge, G. R., Fowler, W. A. \& Hoyle, F., Rev. Mod. Phys. 29, 547 (1955)

[2] Farouqi, K., et al., Astroph. Journ., 712, 1359 (2010)

[3] Nishimura, N., et al., Astroph. Journ. Lett., 836, 21 (2017)

[4] Kasen, D., et al., Nature, 551, 80 (2017)

[5] Cristallo, S., et al., Astroph. Journ., 801, 53 (2015)

[6] Bao, Z.Y. \& Kaeppeler, F., Atom. Data Nucl. Data Tables, 76, 70 (2000)

[7] Guerrero, C., et al., Eur. Phys. J. A, 49, 27 (2013)

[8] Cristallo, S., et al., Astroph. Journ., 696, 797 (2009)

[9] Straniero, O., et al., Nucl. Phys. A, 777, 311 (2006)

[10] Trippella, O., et al., Astroph. Journ., 837, 41 (2017)

[11] Massimi, C., et al., Phys. Lett. B, 768, 1 (2017)

[12] Gallino, R., et al., Astroph. Journ., 497, 388 (1998)

[13] Busso, M., et al., Ann. Rev. Astron. Astrophys., 37, 239 (1999)

[14] Cristallo, S., et al., Nuovo Cimento B, 122, 6 (2007)

[15] Cristallo, S., et al., Astroph. Journ. Suppl., 197, 2 (2011)

[16] Cristallo, S., et al., Astroph. Journ., 833, 181 (2016)

[17] Cristallo, S., et al., Astroph. Journ. Suppl., 219, 21 (2015)

[18] Battino, U., et al., Astroph. Journ., 827, 30 (2016)

[19] Piersanti, L., et al., Astroph. Journ., 774, 98 (2013)

[20] Trippella, O., et al., Astroph. Journ., 818, 125 (2016)

[21] Roederer, I., et al., Astroph. Journ., 742, 27 (2011)

[22] Yong, D., et al., Astroph. Journ., 689, 1031 (2008)

[23] Straniero, O., et al., Astroph. Journ., 785, 77 (2014)

[24] Shingles, L.., et al., Astroph. Journ., 795, 34 (2014)

[25] Prantzos, N.., et al., Astron. \& Astrophys., 234, 211 (1990)

[26] Chieffi, A. \& Limongi, M., Astroph. Journ., 764, 21 (2013)

[27] Pignatari, M., et al., Astroph. Journ., 710, 1557 (2010)

[28] Prantzos, N., et al., MNRAS, 476, 3432 (2018)

\footnotetext{
${ }^{2}$ Note that any nucleosynthesis occurring during the core C-burning phase will not produce chemical variations in the ejecta, because such a material will collapse in the central remnant (being a neutron star or a black hole).
} 